\title{
An Assessment of the Literature and Recent Developments on the Shallow Wake in an Open Channel Flow
}

\author{
Arindam Singha ${ }^{1}$ and Ramaswami Balachandar ${ }^{2}$ \\ ${ }^{1}$ Mechanical Engineering Department, Texas A\&M University at Qatar, P.O. Box 23874, Doha 77844, Qatar \\ ${ }^{2}$ Civil Engineering Department, University of Windsor, Windsor, ON, Canada N9P 3B4 \\ Correspondence should be addressed to Arindam Singha, arindam.singha@tamu.edu
}

Received 15 August 2010; Accepted 10 December 2010

Academic Editor: E. J. Sapountzakis

Copyright ( 2010 A. Singha and R. Balachandar. This is an open access article distributed under the Creative Commons Attribution License, which permits unrestricted use, distribution, and reproduction in any medium, provided the original work is properly cited.

\begin{abstract}
Although shallow flows are very abundant both in nature and in flows of technological importance, their effective characterization started only recently. This type of flow, being vertically confined, exhibits some unique features, which are absent in conventional unconfined flows. A brief review of the related research articles indicates that the exploration of the shallow wake had occurred in three distinct chronological phases. Initially, almost all investigations were aimed at exploring the differences between shallow flows and unconfined flows. Given the differences, the second phase of investigations was mostly concerned with identifying the sources of these differences found already. The third and most recent group of investigations attempted to correlate the flow topology with the extent of the confinement and focused on more applied areas like control of wake instability and consequent effects on drag reduction, and the effect of the coherent structures on species transport. The present paper is intended to present retrospect of the seminal investigations regarding shallow flows, and summarizes the key results from widely ranging studies. Another motivation of the present paper is to provide a basis for future research of shallow wake.
\end{abstract}

\section{Introduction}

Numerous flows occurring in nature can be regarded as shallow [1]. In these flows, the length scale in the horizontal direction is much greater than that in the vertical direction [2]. Examples of shallow flow can be found in rivers, headlands, estuaries, stratified lakes, and coastal seas [1]. Heat dissipated from the earth can bring forth density variation in the vertical direction which can induce shallow flow over mountains and hills [3]. Knowledge of the characteristics of shallow flow and its transport capacity is crucial in modelling and predicting the flow, besides assisting in analyzing the dispersion of heat, pollutants, and biological species. Furthermore, it would also aid in weather modelling as the waterbeds and marshlands play a crucial role in controlling the local weather condition [2].

From the perspective of fluid dynamics, shallow flows display important features due to the presence of the bounding surfaces in the form of the bed and the free surface. The bed imparts vertical shear; while the free surface acts as a stress-free weak boundary. A typical shallow wake can be generated by introducing a disturbance in the form of a bluff body in an otherwise plane flow. The resulting separation of flow from the sides of the body though similar to a deep wake would be modified due to the effect of the imposed boundary conditions. In the subsequent sections, the effects of all these are introduced step by step. Initially, some of the key features of the shallow open channel flow are discussed. This is very crucial since the open channel flow is the background flow for the shallow wake. Then the cause and effect of various factors are discussed parametrically.

\section{Shallow Open Channel Flow}

Open channel flows can be classified as shallow when the vertical length scale of the flow (usually the depth, $H$ ) is significantly smaller than the horizontal/transverse length scales [2]. This definition is based on the kinematic condition, but in turn leads to certain dynamical aspects of the flow. Jirka and Uijttewaal [2] had listed a number of dynamic 
events that can be observed because of the aforementioned constraint. The main complexity of the shallow channel flow arises from the fact that disturbances from the bed may reach the free surface, and vice versa, as a result of vertical confinement. For a conventional deep flow, this interaction is either absent or minimal. Separate quantification (mainly in deep channel flow) of events occurring at bed or at free surface is fairly mature, but the nature of the interaction between these distinct events is not clear enough. The key point of understanding shallow open channel flow may lie in analyzing the nature of this interaction in greater detail.

The near-bed region of a typical channel flow is mainly governed by the wall turbulence produced by the effect of bed shear. This region is highly chaotic, three-dimensional, and a number of dynamical events are found to take place at this region. Numerous studies have been devoted towards exploration of the effect of boundary layer in an effort to understand the associated dynamics of the near-bed mechanisms (e.g., [4-7]). Mainly, the bed region is occupied by negative streamwise fluctuations with fluid particles being lifted away from the bed by positive bed-normal fluctuations (ejection) and positive streamwise fluctuations moving towards the bed (sweep). Above the near-bed region, turbulent bulges of a length scale of the order of two-tothree wall units can be observed [8]. In addition, structures inclined about $45^{\circ}$ to the bed, resembling a horseshoe, appear in the bed, named as hairpin vortices by Theodorsen [9]. Another picture of the near-bed region was put forward by Kline and Robinson [10], which postulates three separate layers of flow close to the bed: low speed streamwise streaks submerged in the viscous sublayer, various flow interaction events corresponding to ejection and sweeps, and above all, three-dimensional turbulent bulges and hairpin vortices. Recent PIV measurements attempt to resolve this discrepancy and found evidence of fluid structures using analysis based on swirling strength, conditional averaging, and two point auto-correlation functions [11, 12]. Lin et al. [13] used proper orthogonal decomposition technique to prove the role of the large-scale structures in the flow features and turbulent structures in the channel flow. Most recently, Roussinova et al. [14] used the same technique on PIV data to expose signature of horseshoe vortex, sweep, and ejections for a typical shallow channel flow case.

The effect of free surface on shallow channel flow was investigated by a number of researchers [15-17], and the general consensus is that the free surface plays an important role in redistributing the turbulent kinetic energy of the flow. Close to the surface, the surface-normal velocity has to diminish to zero, except for the case of waves or flow with inclined surface. The free surface, being stress-free (assuming zero wind-induced stress on the top) boundary, plays a major role in this redistribution process. But a wide range of opinion exists about where the redistribution takes place. Hunt and Graham [18] indicated that the redistribution takes place in two separate regions directly beneath the free surface: the outer source region and the inner viscous region. According to them, the normal to surface component of turbulence decreases and the surface-parallel component increases inside the outer layer. The thickness of the outer layer is of the order of the integral length scale. The inner layer lies in between the outer layer and the free surface and the vertical velocity rapidly decays to zero to the free surface within this layer. Walker et al. [19] estimated the thickness of these two layers as approximately one and one-tenth of the turbulent integral length scale. Contrary to the opinion of Hunt and Graham [18], they indicated that the energy redistribution takes place only in the large-scale structures of the flow, but the small-scales remain unaffected. However, experimental evidence put forward by Balachandar and Patel [20] shows that for low turbulent intensity, the whole flow field can be affected by the boundary condition imposed by the presence of the free surface. They concluded a change in the Cole's $\Pi$ parameter as well as rate of production of turbulent kinetic energy due to the presence of free surface disturbance.

Like the near-bed region, it is equally important to identify and characterize the fluid structures associated with the free-surface dynamics, and a number of studies are directed towards this objective. At this point, it must be noted that investigation of near-surface structures can be very difficult experimentally (appearance of surface waves, bubbles) as well as computationally (lack of proper model). Generally speaking, close to the free surface mainly two types of structures can be observed: surface-normal vortex tubes with predominantly vertical velocity component and surface-parallel vortex tubes buried inside the surface layer. Physically, it appears relevant to assume a direct linkage between the surface normal vorticity, and the surface undulations via the "pressure" exerted by the vorticity patch. Dommermuth [21] measured the free surface deformation, and vorticity magnitude simultaneously to conclude the existence of a rather weak correlation between them, and it was supported experimentally by Zhang et al. [22]. But Weigard and Gharib [23] used simultaneous shadowgraph and PIV results to show that the location of the peaks of vertical vorticity matches well within range with the location of free surface deformation. Along the same line, efforts were made to computationally examine the free surface flow structures, and the first direct simulation study was reported by Lam and Banerjee [24]. Worth mentioning are the studies of Komori et al. [25] and Handler et al. [26], which used a full free surface model unlike the case of Lam and Banerjee [24] who used a rigid-lid assumption. The most important and comprehensive study was carried out by Pan and Banerjee [27] which classified the structures into three distinct groups: vortices, upwelling, and downdraft. Signatures of these types of structures were also documented experimentally by Gupta et al. [28], but they did not effectively classify them. "Vortices" were defined as the region of cluster of surfacenormal vorticity, where the ratio of surface-normal to the surface-parallel velocity is approximately zero. "Upwelling" was defined as the region of divergence of streamlines with large surface normal velocity. "Downdraft" is the region, which helps to nullify the effect of the upwelling and exhibit strong downward flow component from the free surface. These three types of structures were found to appear in a certain pattern: typical upwelling reaches the free surface and interacts to generate a donut-shaped vorticity pattern 
there. Upon interacting, these patterns generate a number of short-lived smaller vortices around the edge of the upwelling. A typical pattern concludes with the downdraft to wash away the excess fluid close to the free surface. The energy spectra from the locations close to the free surface show $k^{-(5 / 3)}$ and $k^{-(7 / 3)}$ region, which are characteristic of twodimensional turbulence and indicate occurrence of turbulent kinetic energy redistribution. However, at a distance from the free surface, usual characteristics of three-dimensional turbulence prevail.

Note that it is the unique nature of the interaction of these events originating from the bed and free surface that makes the shallow channel flow distinctive from the conventional open channel flow. As a result of this interaction, a clear separation of scales can be observed. Jirka and Uijttewaal [2] has aptly summarized the following: "Shallow flows are largely unidirectional, turbulent shear flows driven by piezometric gradient and occurring in a confined layer of depth. This confinement leads to the separation of scale, three-dimensional turbulence $l_{3 D} \leq H$, and large scale twodimensional turbulent motion, $l_{2 D} \geq H$, with some mutual interaction." Two plausible but much generalized solutions were provided by Nikora et al. [29] and Shen et al. [30]. According to Nikora et al. [29], due to smaller vertical extent of the flow, events from the bed reach the free surface and vice versa. When three cylinder-like fluid structure becomes sufficiently long to reach the free surface, they appear as smaller tornado-like vertical cylinders submerged in the channel flow. The complex interaction between these cylinders with the flow generates these two-dimensional structures. Shen et al. [30] indicated that the hairpin eddies from the bed may reach the free surface and, when the head part dissipates away due to the convection effect of the mean streamwise flow, and the remaining leg part of the hairpin vortex gives rise to these two-dimensional structures.

\section{Shallow Wake}

Shallow wake has basically the same general characteristics of a deep wake. Upon accroaching a bluff body, a stagnation point along the upstream edge occurs with boundary layer along the wall. When the flow encounters sudden change in geometry, of an adverse pressure gradient, separation occurs from the sides of the body, giving rise to a von Karman vortex street. A detailed description of the deep, conventional wake can be found in Zdravkovich [31]. Whereas the overall broad flow picture remains the same, shallow flow imposes a number of different characteristics, which are crucial for the overall development of the flow features. First, the limited depth would restrict the onset of three-dimensional breakdown of the vortex street. As a result, the von Karman vortex street can be observed for a larger range of Reynolds number in a shallow wake. For example, the pattern of crude oil spillage from the ship Argo Merchant stranded at Nantauket shoals in Massachusetts shows a clear vortex street pattern at a very high Reynolds number $\operatorname{Re} \approx 10^{7}$ [32]. Secondly, the bed friction tends to arrest the transverse growth of the disturbances associated with a typical vortex street pattern and stabilize the wake. This may occur either in the near-wake or in the far-wake region. For the case of a shallow wake, the stability number, as will be shown later, determines the effect of the bed friction and the subsequent wake stability. In the near wake region, if the stability number $S_{\text {near }}=C_{f} D / H$, where $C_{f}$ is the skin friction coefficient, $D$ and $H$ are the characteristic diameter of the body and flow depth, respectively, exceeds a critical value, wake stabilization takes place. In the far-wake region, stability number is defined as $S_{\mathrm{far}}=C_{f}(2 b) / H$, where $b$ is the local wake width. Similar wake stabilization takes place at the far wake region if $S_{\text {far }}$ exceeds a certain critical value.

3.1. Stability Number. Crucial to the development of the shallow wake is the mutual interaction of the vertical shear due to the approaching flow with the horizontal (transverse) shear due to the variation of momentum deficit in the transverse direction. Therefore, the spatiotemporal growth of the shallow wake is controlled by their mutual interaction. The shallow wake is maintained in an equilibrium state as a balance of two different effects: on one hand, turbulent kinetic energy is extracted from the mean flow and is fed to the large-scale coherent structures by interaction with the lateral shear layer. On the other hand, in the bottom boundary layer, the turbulent kinetic energy is extracted from the large-scale structures and dissipated through the small-scale structures according to Kolmogorov theory of local isotropy. Thus, one can balance the loss of the turbulent energy due to the bottom friction by the production of the turbulent kinetic energy by the lateral shear layer. The energy fed into the large-scale coherent structures can be computed as the product of the coherent velocity fluctuations and the transverse mean streamwise velocity gradient as [33],

$$
P=\left\langle u_{c} w_{c}\right\rangle \frac{\partial U}{\partial z},
$$

where the subscript " $c$ " denotes the velocity associated with the coherent motions only. $u$ and $w$ indicate the instantaneous velocity in streamwise and transverse directions, respectively. Further, $x$ - and $z$-denote the streamwise and transverse directions, respectively. The produced energy is dissipated through the small-scale fluctuations at the bottom boundary layer. Babarutsi and $\mathrm{Chu}$ [33] showed that if the velocity associated with the coherent structure is small compared to the mean flow, then the dissipation can be approximated as

$$
F_{b}=\frac{C_{f}}{2 H} U\left(2\left\langle u_{c} u_{c}\right\rangle+\left\langle w_{c} w_{c}\right\rangle\right),
$$

where $C_{f}$ is the skin friction coefficient and $H$ is the depth of flow.

Dividing the above two equations, the flux stability number can be approximated as

$$
S_{\text {flux }}=\frac{F_{b}}{P}=\frac{C_{f} U}{2 H(\partial U / \partial z)} \frac{2\left(\left\langle u_{c} u_{c}\right\rangle+\left\langle w_{c} w_{c}\right\rangle\right)}{\left\langle u_{c} w_{c}\right\rangle} .
$$

The ratio on the right represents the ratio of the coherent Reynolds normal and shear stress, respectively. Chu et al. [34] 
proposed a gradient stability number for shallow flow, which has an inflexion point in the transverse distribution of the streamwise velocity, that is, $\partial^{2} U / \partial z^{2}=0$. Without affecting the calculation, the term at the extreme right can be dropped safely and the velocity gradient can be expressed as $\Delta U=$ $U_{1}-U_{2}$, where $U_{1}$ and $U_{2}$ are characteristic velocities on each side of the shear layer of local thickness $\delta$. Thus, the gradient stability number

$$
S_{\text {grad }}=\left.\frac{C_{f}}{H} \frac{U}{\partial U / \partial y}\right|_{\text {Inflexion }} \approx C_{f} \frac{\delta}{H} \frac{U_{\mathrm{avg}}}{\Delta U}=\frac{C_{f}}{2 R} \frac{\delta}{H},
$$

where

$$
R=\frac{U_{1}-U_{2}}{U_{1}+U_{2}}=\frac{\Delta U}{2 U_{\mathrm{avg}}} .
$$

This equation is applicable for any general shear layer. For the case of a shallow wake behind a bluff-body, it can be applied at the lee end of the recirculation bubble. Since $U_{2} \approx 0, R=$ 1. The width of the shear layer is approximated to be half of the characteristic diameter of the body $(D / 2)$. Using these, the wake gradient stability number can be found as

$$
S_{\text {grad }}=\frac{1}{4} C_{f} \frac{D}{H}
$$

The wake stability number $(S)$ is defined as four times the gradient stability number for sake of convenience,

$$
S=4 S_{\text {grad }}=C_{f} \frac{D}{H} .
$$

Unlike the gradient stability number, stability number $(S)$ is a global parameter, and it does not depend on the choice of local coordinate system. Therefore, it is a more universally accepted number and mentioned extensively in shallow wake literature.

3.2. Effect of the Bed. As mentioned earlier, the uniqueness of a shallow wake depends on the combined effect of the bed and the free surface. Although these two effects are closely interrelated, it may be possible to view them independently. Wolonski et al. [35] defined an island wake parameter, $P_{\text {island }}=U_{a} H^{2} / k_{z} D$, where $U_{a}$ is the ambient flow velocity and $k_{z}$ is the vertical eddy. The vertical eddy viscosity was approximated as $k_{z}=0.15 H U_{\tau}=0.15 H U\left(C_{f} / 2\right)^{1 / 2}$, where $U_{\tau}$ is the friction velocity. They have suggested that a critical value of $P_{\text {critical }}$ of the order of unity exists, and a vortex street type of wake is not possible for $P_{\text {island }}<P_{\text {critical }}$. Their classification is somewhat incomplete and lags descriptive information, as they are based on satellite imaging and temperature mapping of the island wake and no direct measurements were conducted to bolster the classification.

An important investigation in shallow wake was performed by Ingram and Chu [36] by observing the oceanic shallow wake behind Rupert Bay, Ontario. Initially, they formulated an analytical description of the effect of the bed on the shallow flow. To complement the formulation, they performed flow visualization in the laboratory. A total of six laboratory observations and 26 events of oceanic shallow wakes at Reynolds numbers $(\operatorname{Re}=4 H U / v)$ ranging from 4,700 to 11,000 , and stability numbers ranging from 0.03 to 0.65 have been reported. They introduced the stability number as a measure of the bottom friction effect on the shallow wake. Based on a series of observations, they postulated a critical stability number of 0.48 , above which the oscillating characteristic of the wake gets stabilized due to the bottom friction. Below the critical stability number, the effect of bed friction is not strong enough to stabilize the wake and vortex street type of wake can be observed. Figure 1 is adopted from the paper and depicts three different cases; (a) $\operatorname{Re}=11,000, S_{w}=0.031$, (b) $\operatorname{Re}=11,000, S_{w}=0.013$ and (c) $\operatorname{Re}=4,700, S_{w}=0.054$. Clearly seen in this figure is the suppression of the wake oscillation with change in the Reynolds number and stability number. The frictional effect of bed is also shown to affect the entrainment coefficient of the wake. However, the range of Reynolds number and the stability number for the investigation is not large enough to draw firm conclusions about the state of the wake.

Along the same lines, extensive laboratory experiments (57 with circular cylinder, 54 with solid plate, and 34 with porous plate) and visualization of shallow wake were performed by Chen and Jirka [37] covering a wide range of Reynolds number and stability number $\left(\operatorname{Re}_{h}=570-7,300\right.$, $\left.\operatorname{Re}_{d}=3,000-4,14,000, S=0.01-0.94\right)$. Based on visual observations, they divided the shallow wake into three different categories: vortex street type (VS), unsteady bubble wake (UB), and steady bubble wake (SB). If the perturbation due to the separating shear layer is dominant, the shallow wake resembles the well-known von Karman vortex street as seen in a conventional deep wake. Although qualitatively similar, the vortex street of shallow wake and the conventional von Karman vortex street have differences too. The vortex street (VS) shows a streaky and fuzzy appearance due to the presence of the small-scale structures arising from the bed frictional effect. This fuzziness sometimes causes blurring out of the well-observed roll up of the vortices. This type of wake is visible for $S \leq 0.2$. If the stability number is increased $(0.2 \leq S \leq 0.5)$, the bed friction effect increases and the flow separates from both sides of the body simultaneously, not alternatively. A nearly steady bubble is formed immediately downstream of the body. But the end of the bubble oscillates alternatively and this leads to periodic breakdown of the vortices. This is called the unsteady bubble (UB) wake. The steady bubble (SB) mode can be observed when the bed friction effect decreases the momentum to such an extent that the recirculation bubble stays almost stable at the lee of the body and no shedding is observed. By analytical approximation of the wake momentum deficit, Chen and Jirka [37] defined a length scale $L$ beyond which the wake momentum deficit disappears

$$
L=\frac{2 H}{C_{f}}=\frac{2 D}{S} .
$$

Unstable shallow wakes get stabilized after a streamwise distance of the order of $L$, mainly due to the increasing momentum deficit, and subsequent increase in the stability parameter. They also observed that the width of the shallow wake greatly exceeds the depth of the flow, and as a result the 


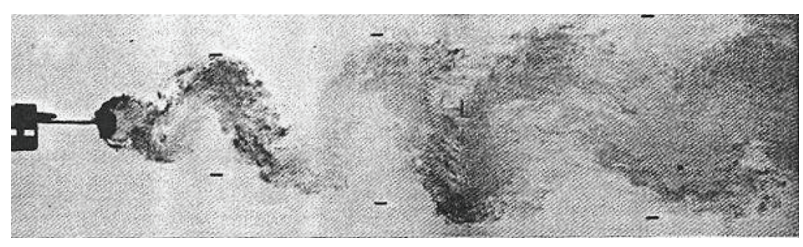

(a)

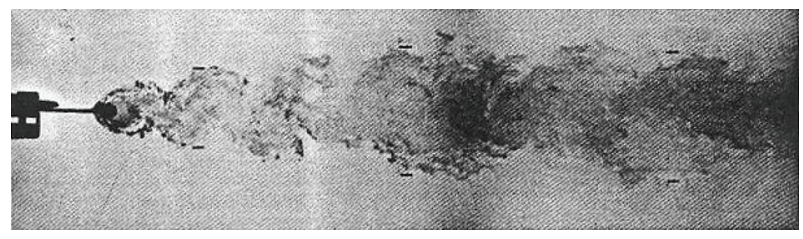

(b)

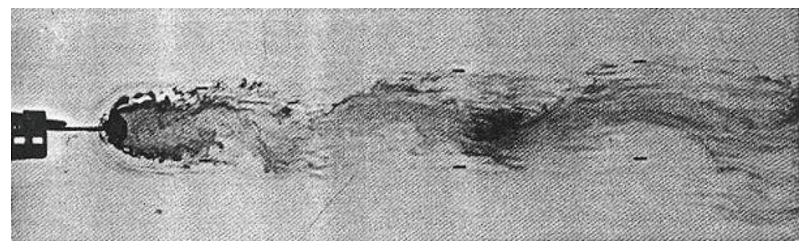

(c)

FIGURE 1: Qualitative demonstration of the effect of the bed on the development of shallow wake (Reproduced with the permission of American Geophysical Union).

flow structures are mainly two-dimensional in nature. They suggested that the majority of the effect of the bed friction is agglomerated at the bottom boundary layer and may not contribute towards the three-dimensionality of shallow flow. The bed friction effect is averaged over the full depth of flow and is manifested in the form of the wake stability number.

Negretti et al. [38] mounted artificial roughness mats on the bed of a shallow wake flow to investigate the effect of the increased roughness. Two series of experiments were conducted; for the first series, roughness elements were placed in the lateral shear layer region and for the second series, the roughness elements were placed at the end of the recirculating bubble. For the first case, roughness was found to decrease the magnitude of the Reynolds stress in addition to narrowing the wake width. Also, the wake behaved very similar to a stable bubble wake, since the introduced roughness reduces the momentum exchange with the ambient flow. When the roughness element was placed at the back of the re-circulating bubble, the usual wake behaviour was observed till the flow reached the roughness and the fluid structures were destroyed while flowing over the roughness. This study explicitly indicates that the bed roughness has the ability to alter the near-wake as well as the far-wake structure. Mainly these three studies investigated the effect of bed friction only, and explicitly, although a number of investigators examined the effect of bed friction as a part of a study intended to look into some other aspect of the shallow wake.

3.3. Effect of Free Surface. Quantification of the effect of the free surface is always difficult both computationally and experimentally. Computationally speaking, until recently there was no proper model available for simulating the free surface other than imposing the rigid-lid assumption on it. Experimentally speaking, using a laser is very difficult because of the wavy characteristics of the free surface and the appearance of the bubbles. Despite these difficulties, a number of studies were performed in this direction and were mainly concerned about exploring the relationship between the development of free surface deformation and turbulent anisotropy [17, 19, 39-41].

Logory et al. [41] used LDV and PIV to investigate the effect of free surface on an otherwise deep wake. Due to the large water depth, the effect of bed is expected to be confined close to the bed and not penetrate beyond a certain vertical distance from the bed. As a result, close to the free surface, the sole effect of free surface will be realized with reduced contamination arising from bed friction. In fact, LDV measurement of the wake flow behind the bluff body, measured at half-depth, matches well with the published data for the deep wake. But close to the free surface (depth $12 \sim 13 \mathrm{~mm}$ below), the wake width was found to be doubled in size, while the centerline velocity remained almost constant. The contour of the mean streamwise velocity in the streamwise plane downstream of the body, adopted from Logory et al. [41] is shown in Figure 2. The self-similar behavior of the mean and root-mean-square velocity is also lost at the free-surface location. PIV measurements at a depth $\sim 20 \mathrm{~mm}$ below the free surface showed evidence of "upwelling" and "downdraft" as seen in a shallow flow, in addition to the large-scale structures originating due to the presence of the body. At the near surface layer, opposite signed streamwise vorticity was found to exist in pairs at both sides of the wake centerline, which induces flow from the centerline towards the ambient flow region. In the deep wake, vortex structures are predominantly two-dimensional, but close to the surface, the vortex structures tend to reorient themselves in either surface-normal or surfaceparallel direction. The magnitude of the surface normal vorticity was found to be smaller compared to the deep wake region. The origin of these structures is believed to be the quasi-streamwise rollers originating from the turbulent boundary layer on the bluff body. The existence of these surface-normal structures was also confirmed by the LIF (laser induced fluorescence) flow visualization. A histogram of the vorticity of the surface-parallel structures revealed that they are inclined at $45-50^{\circ}$ angles from the wake centerline and induce a velocity away from the wake centerline directed to the upstream direction. The resulting motion from this complex vorticity distribution causes the wake widening at the near-surface region as the surface-parallel structures carry low momentum fluid towards the ambient flow region.

Based on the available literature and his own analysis, Maheo [17] summarized the near surface dynamics of a typical shallow flow, for the case of turbulent jet and wake. He found that the free surface only affects the velocity components in a thin layer close to the surface. The thickness of this surface layer was approximately either onehalf of the local width or the vorticity thickness of the wake or jet, respectively. Inside this layer, the fluctuation 


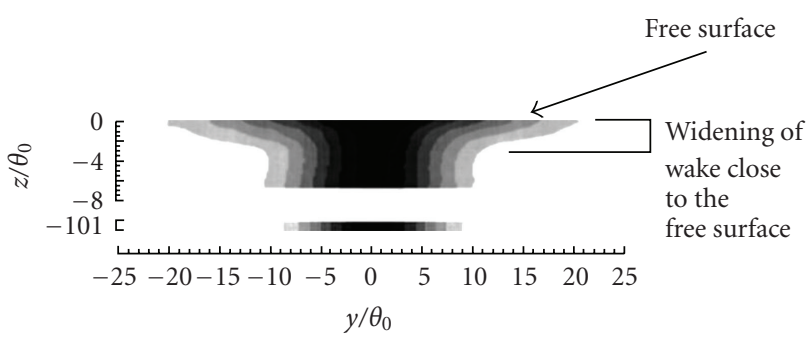

FIgURE 2: Widening of the wake close to the free-surface (Adopted from Logory et al., [41]). The contour color represents mean velocity; white to dark shade represents higher to lower range of streamwise velocity. $\theta_{0}(=3.1 \mathrm{~mm})$ is the half-momentum thickness of the wake (Reproduced with the permission of American Institute of Physics).

in the vertical direction gets retarded and the turbulent kinetic energy in the vertical direction was redistributed to the horizontal velocity components in the streamwise and transverse directions. The streamwise vorticity tubes buried inside the surface layer were found to generate surface currents which affect the dynamics of flow. Figure 3 shows a typical signature of the surface currents close to the free surface. The motions of these structures contribute towards widening of the surface shear flows, compared to the deep flow case. He found that the widening of the turbulent shear layer at the near surface can be as much as $20 \%$ for a turbulent wake and $25 \%$ for a turbulent jet.

3.4. Effect of Approaching Velocity. The incoming open channel flow approaches and senses the presence of the body. When the approaching flow meets the body, a vertical stagnation line is generated and the total flow divides along the upstream sides of the body. The resultant stagnation pressure at the body at a certain elevation is higher than at an elevation closer to the bed due to the nonuniform incoming velocity profile. Consequently, a vortex tube is generated and elongated along the sides of the body, due to the accelerating flow region (Region III, [31]) at the sides of the body. This particular system of vortex tube is called the horseshoe vortex, and it plays a crucial role in the development of shallow wake. Figure 4 illustrates a typical flow model of shallow wake. The sign of vorticity of the approaching flow as well as the horseshoe vortex is shown. Due to the complex interaction process of the bed and free surface with the flow structures, a shallow wake has a considerable threedimensionality associated with it.

Rao et al. [42] investigated the effect of the approaching velocity on the bed-structure interaction for a typical shallow wake by a qualitative dye visualization study for flow depths of 150,200 , and $250 \mathrm{~mm}$, respectively. The maximum streamwise velocity and Reynolds number ranges were $U=$ $0.08-0.21 \mathrm{~m} / \mathrm{s}$ and $\mathrm{Re}_{d}=1500-4400$, respectively. Dyes of different colors were injected into the flow through ports situated at different elevations on the cylinder. Figure 5 shows the side view of a circular cylinder, immersed in a shallow channel flow of $100 \mathrm{~mm}$ depth. The red dye from the lower port gets entrapped into the horseshoe vortex and followed the vortex pattern closely. The blue dye

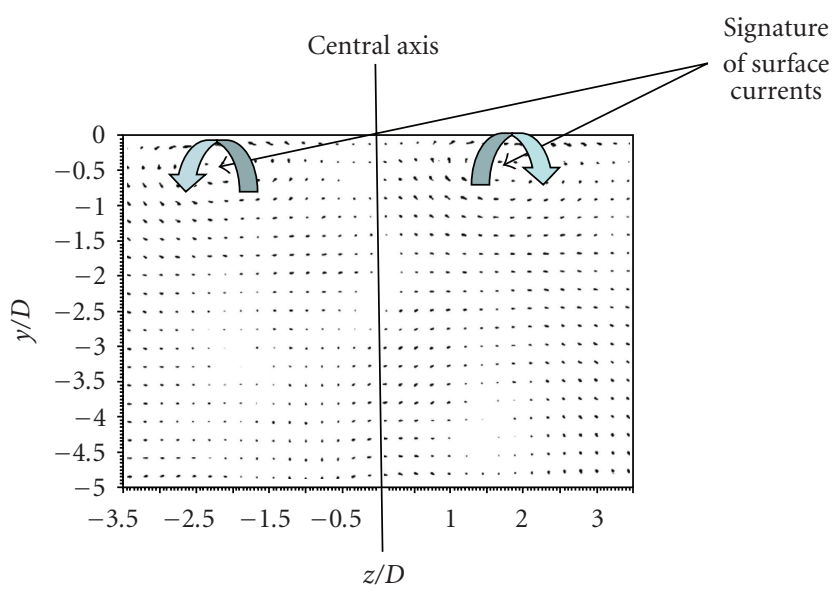

Figure 3: Signature of the surface currents as seen in a plane normal to the streamwise direction downstream of a bluff body. (Reproduced by permission from Maheo).

follows the vortex shedding from the side of the body. A distinguishing feature of the horseshoe vortex is the upward three-dimensional rollup of the horseshoe vortex and the subsequent interaction with the vortex street emanating from the sides of the body. This interaction leads to the three-dimensionality and vertical variability of the shallow wake. Amongst different conclusions, they found a vertical oscillation of the shear layer at the sides of the body, the source of which was tracked back to the horseshoe vortex wrapping around the body. As mentioned earlier, they connected the dynamics of the horseshoe vortex to be the possible source of the three-dimensionality of the shallow wake. However, the interactions needed to be quantified.

Wang et al. [43] investigated the effect of variable momentum thickness of the approaching boundary layer on different aspects of the downstream wake flow. The momentum thickness of the approaching boundary layer was adjusted to be $0.07 D, 0.13 D$, and $0.245 D$, where $D(=20 \mathrm{~mm})$ is the characteristic width of the body. The Reynolds number of the flow was $\operatorname{Re}_{D}=11,500$. In this experiment, the flow was allowed to move over the top of the body, that is, the body does not pierce the free surface. This does not really comply with the standard definition of a shallow wake, but some of the conclusions may be worth mentioning. With increasing momentum thickness of the approaching boundary layer, an upward flow originating from the bed and towards the free surface was noticed. This upward flow contributed towards the reduction of the reverse flow region close to the bed. At the same time, the magnitude of the Reynolds stress decreased close to the bed. Also, the variable thickness of the approaching flow was found to alter the nature of the vortex shedding from the body (symmetric/anti-symmetric). These studies indicate that the approaching flow has a significant influence on the shallow wake flow.

3.5. Two-Dimensional Coherent Structures of the Shallow Wake. The turbulent channel flow can be disturbed due to several reasons which can result in oscillation and instability 


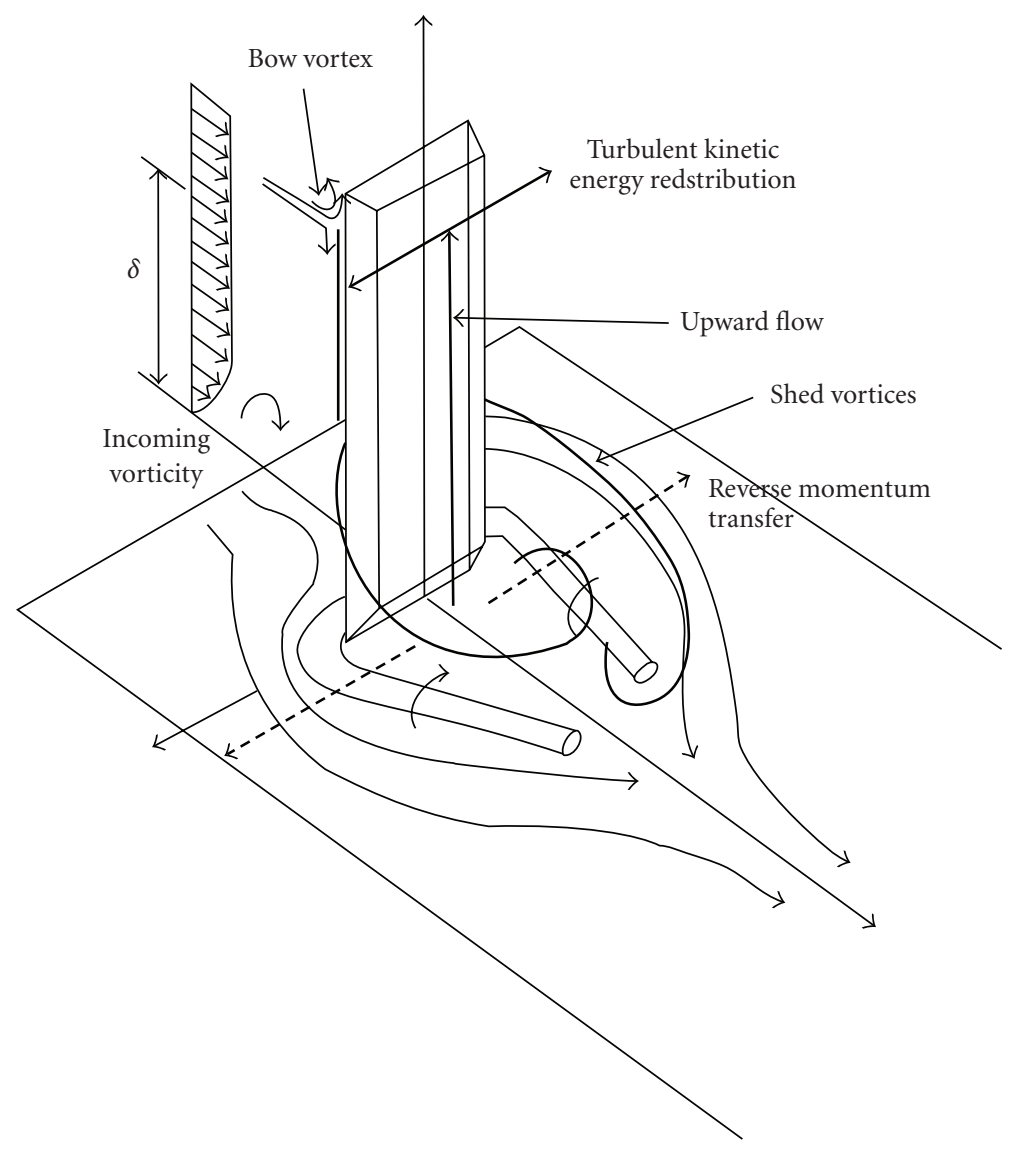

FIgURE 4: A simple model of the shallow wake, as constructed from the available literature.

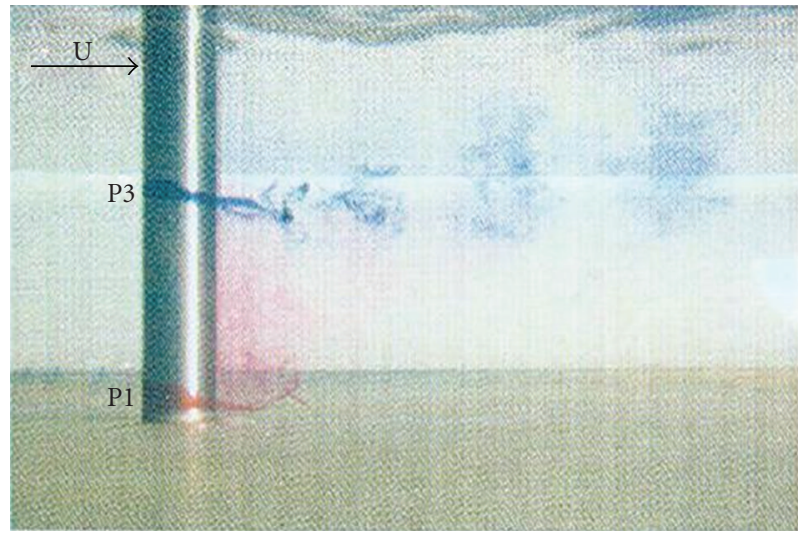

Figure 5: Qualitative demonstration of the dynamics of horseshoe vortex around a circular cylinder as the source of threedimensionality of shallow wake (Reproduced by permission from Visualization Society of Japan).

of the flow. If the strength of these instabilities sometimes exceeds a certain threshold, this can grow in space extracting energy from the mean flow and grow in size. During the process of growth, sometime, the transverse size of these eddies may exceed the vertical flow depth. Due to purely kinematic reason, the vorticity vector of these eddies, termed as two-dimensional coherent structures (2DCS), must be perpendicular to the bed or the free surface. Of course, smaller coherent structure whose size is less than the depth of flow contains no preferential direction of orientation and exhibits three-dimensional behaviour. The appearance of the 2DCS has been consistently reported by a number of researchers [37, 44-46].

Holmes et al. [47] and Bonnet et al. [48] reviewed the recent literature on the importance and the characteristics of coherent structures in different kinds of turbulent flow. Although there is no general consensus about the definition of a coherent structure, Hussain [49] formed a working definition as follows: "A coherent structure is a connected (large-scale) turbulent fluid mass with an instantaneously phase-correlated vorticity over its spatial extent". Carmer and Jirka [50] showed that the transport capacity of these largescale structures is about two-orders of magnitude higher than the small-scale structures. Therefore, characterization of the large-scale coherent structures will lead to a better modeling of discharge and dispersion of pollutants released in shallow rivers or lakes.

Jirka [51] has indicated three different types of forcing mechanisms for the generation of the large-scale coherent structures:

Topological Forcing (Type A). Presence of a body (e.g., island, headlands, jetties, etc.) in a shallow flow represents the strongest mechanism for generation of 2DCS. With development of these eddies, the interaction between them and the 
bed increases. As a result, more energy is extracted from the mean flow and is fed to the rotational energy of the eddy. In this case, turbulent kinetic energy flows from the smaller to the larger scale and therefore exhibit inverse energy cascade.

Internal Shear Flow Instability (Type B). Varying momentum flux (deficit or excess) due to the velocity gradient in the lateral direction may trigger this type of instability. Such triggering action can be caused by the presence of a source flow like shallow jet, wake, or mixing layer.

Secondary Instability of the Base Flow (Type C). This is the weakest type of instability and experimental evidence is sparse (except for [29]). Consider the case of a shallow channel flow containing a vertically sheared boundary layer and 3D turbulent coherent structures. This flow will be maintained in equilibrium by the bed friction. However, a slight imbalance in the flow will lead to the redistribution of the turbulent kinetic energy in the entire range of wave numbers. Supercritical flow (inertia dominated gravity forces) gives a 2D turbulence inverse cascade, while subcritical flow (gravity dominates inertial force) gives direct cascade of energy. The distortion of the vortex line caused by the secondary instability will lead to the formation of 2DCS provided the magnitude of the disturbance exceeds a certain critical level.

Following the work of Dracos et al. [44] and Uijttewaal and Tukker [45], the generation and development of the 2DCS can be divided into a number of stages in time and space. The "near-field" of any shallow flow is characterized mainly by the two-dimensional transverse shear layers and contains small-scale three-dimensional structures. The "middle-field" has strong three-dimensionality because of the interaction of the bed and free surface. At the "far-field", the structures generated earlier grow in size greater than the depth of flow. To preserve the kinematic condition, these eddies must have two-dimensional character and will have dominant vorticity vector perpendicular to the free surface. Also, the mean flow, whose transverse scale is of the same order as the large turbulent eddies, became approximately two-dimensional.

Following the generation, the 2DCS grows and develops by entraining outside ambient fluid. In addition to this, two or more 2DCS may merge together to generate a larger 2DCS [45]. It must be mentioned here that the limited depth restricts the mechanism of vortex stretching that is present in three-dimensional turbulent flow. Merging of 2DCS to form larger 2DCS leads to the flow of turbulent kinetic energy from the high to the small wavenumber range and therefore exhibits inverse energy cascade [52]. The possible existence of the two-dimensional turbulence in shallow flow was demonstrated by Dracos et al. [44] and Chen and Jirka [37] by computing the velocity spectra. The 2DCS lose their energy owing to the effect of the bed friction. Dimensionally, it can be shown that an eddy of size $2 H / C_{f}$ will loose all its energy in one rotation due to bed friction [50].

3.6. Other Related Literature and Vertical Variability of Shallow Wake. There are other studies in the literature related to the shallow wake. These include, for example, searching for suitable scale to absorb the effect of bounding surfaces, and investigation of the variation of the flow characteristics as a function of the vertical elevation from the bed. Balachandar et al., [53] classified the shallow wake into shallow-shallow and deep-shallow wake based on visual observation and video-imaging of dye concentration injected in shallow wake. The depth of flow, mean free stream velocity, and Reynolds number were varied between $10-40 \mathrm{~mm}, 76-157 \mathrm{~mm} / \mathrm{s}$, and 1570-4810, respectively. When the stability number was lower than a critical value $\left(S_{w}<0.008\right)$ then the von Karman type vortex street was observed. For stability numbers greater than the critical value, either intermittent vortex street was observed or was completely annihilated. This classification is more like that of Wolansky [54] for island wake as discussed earlier. Figure 6, adapted from Tachie [55], shows an example of the deep-shallow and shallow-shallow wake, respectively. The left figure corresponds to higher depth of flow $(H / D=$ 2.00), and the right figure corresponds to lower depth of flow $(H / D=0.25)$. The vortex street can be seen for the case of higher depth of flow, but it is completely annihilated for lower flow depth due to increased bed friction effect. In a later study to characterize turbulent shallow wake, Balachandar et al., [56] performed velocity measurements downstream of a flat plate immersed in shallow channel flow. Measurements were carried out at three different vertical locations, $H / 4, H / 2$, and $3 H / 4$. Several normalizing parameters were attempted in an effort to collapse the mean streamwise velocity defect at different streamwise locations and depth of flow. $C_{d} \delta_{v}$ was identified as one of the proper normalizing length scales, where $C_{d}$ and $\delta_{v}$ are the drag coefficient and the half-width of the wake, respectively. At a given distance from the bed, the approaching velocity upstream was found out to be one of the normalizing scales for streamwise velocity deficit, for which the data collapse fairly well onto a single profile for all streamwise and vertical measurement locations. It should be mentioned here that for the deep wake, the approaching velocity is constant with vertical distance from the bed, whereas for shallow flow, it is a function of the vertical distance. A friction parameter $\beta$ was proposed to represent the relative effect of the bed friction and transverse shear. Above a critical value, $\beta_{c}=0.2$, the organized motion of the vortices began to disappear.

To quantify the effect of the bed roughness, Tachie and Balachandar [57] measured velocity distribution of a shallow wake generated on a smooth and a rough wall. The roughness was found to affect both the mean and turbulence parameters of the of the wake flow. For approximately the same value of Reynolds number, the width of the wake was found to be much smaller for the rough bed compared to the smooth bed. Also, the ratio of the transverse shear to the bed friction was found to be smaller for the case of the rough bed. Shallowness of the flow was also found to enhance the turbulent fluctuations in the flow.

In a series of papers, the general features, stability, and control of shallow wake have been further addressed [58-60] using PIV. The experimental procedure for all these investigations is more-or-less similar; a circular cylinder is placed in a shallow channel flow. In the first paper, the focus was on the 


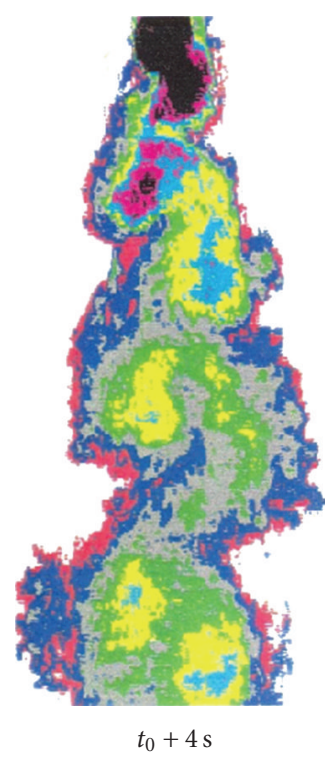

(a)

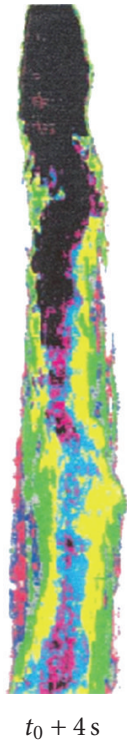

(b)
FIGURE 6: Example of deep-shallow and shallow-shallow wake (Reproduced by permission from Tachie).

spatiotemporal evolution of the vortex formation process. In the second and third investigations, they addressed the effect of Reynolds number on the flow and explored different possible ways to stabilize the wake, respectively. The shallow wake was found to be characterized by large-scale vertical structures (or 2DCS) emanating from the body downstream of the cylinder. However, their investigation discovered an axial flow from the bed towards the free surface through the core of these structures, supporting the finding of Cohn and Koochesfahani [61]. This flow was believed to contribute significantly to the three-dimensionality of the shallow wake structure. The instantaneous streamline topology and vorticity contours at different elevations from the bed compare well, in a general sense, with a conventional deep-wake, but a signature of the three-dimensionality was also observed. The location of the saddle point in the instantaneous streamline topology was found to be a function of the distance from the bed. With increasing distance from the bed, the spanwise extent of the instantaneous vorticity contour as well as the streamline topology grows in the transverse direction, probably due to the diminishing effect of the bed. Close to the bed, a unique structure of the instantaneous streamline was observed, called as owl face of the first kind [62], which has a very well-defined pair of foci and saddle points. With increasing distance from the bed, the organization of the foci as well as the saddle points is lost. At the near-bed location, the vorticity contour was found to be tilted toward the central region, but with increasing distance from the bed, the tendency of tilt decreases. Additionally, the peak magnitude of vorticity was also found to be dissipated rapidly close to the bed, than at some locations far from the bed. The contour of the time-mean Reynolds stress was found to be very well defined in accordance with the shear layer separation, except close to the bed. In the near-bed region, additional cluster of
Reynolds stress was noticed which explains the interaction of the horseshoe vortex with the shear layer at the sides of the cylinder. Unlike the vorticity, the peak magnitude of the Reynolds stress remains almost the same and does not change with the distance from the bed. The stability number of the shallow wake was found to play an important role in the resulting mode of the wake. For example, increasing the stability number leads to the attenuation of the appearance of the large-scale coherent structures in the wake. Despite this, a well-defined varicose instability was observed. The reason of this particular instability can be attributed to the dynamics of horseshoe vortex wrapping of the body. At a even higher stability number, the wake was completely stabilized, but smaller amplitude rotational motions were noticed to set off now and then in the near-wake region. In the third investigation of the aforementioned series, Cohn and Koochesfahani [61] introduced a variable width streamwise slot cut through the cylinder. For smaller slot size, the cluster of vorticity preserved their shape, but appeared downstream compared to the case of no-slot. Also the frequency of undulation of the emanating jet from the slot may affect the shedding frequency of the vertical structures.

At this point, it is obvious that the flow variables of a shallow wake are different at different distances from the bed, and this can be termed as vertical variability of shallow wake. Singha et al. [46] explored the vertical variability of a typical shallow wake in an open channel flow. The stability number of the wake was small (0.0015), and velocity measurements were performed at near-bed, mid-depth, and near-surface vertical locations to reveal the vertical variability. Figure 7 is adopted from the paper and clearly illustrates the difference of the wake structure at different vertical locations. They also identified some key features of the flow: effect of the bed friction to dampen the transverse growth of the wake, existence of sweep and ejection type of events at the free surface contributing towards redistribution of the turbulent kinetic energy at the near-surface location, the effect of the structures at near-bed and free-surface region towards enhanced momentum transfer at these locations. In a continued study, Singha [63] applied POD and closedstreamline based coherent structure identification scheme to detect the location, size, and strength of the coherent structures at near-bed, mid-depth, and near-surface. The finding clearly indicates that the bed as well as the free surface tends to reduce the size and strength of the coherent structures, but the effect is more drastic for the bed rather than the free-surface. The largest and strongest structures can be found at the mid-depth location only. Also, the horseshoe vortex as well as free surface events was found to affect the location of appearance of the coherent structures at near-bed and near-surface vertical locations.

\section{Summary and Future Prospects}

The related literature on shallow wakes in an open channel flow has been reviewed to highlight the effect of three important parameters, namely, the effect of the bed, the effect of the free surface, and the effect of the nonuniform approaching flow. The possible cause and effect of these three 


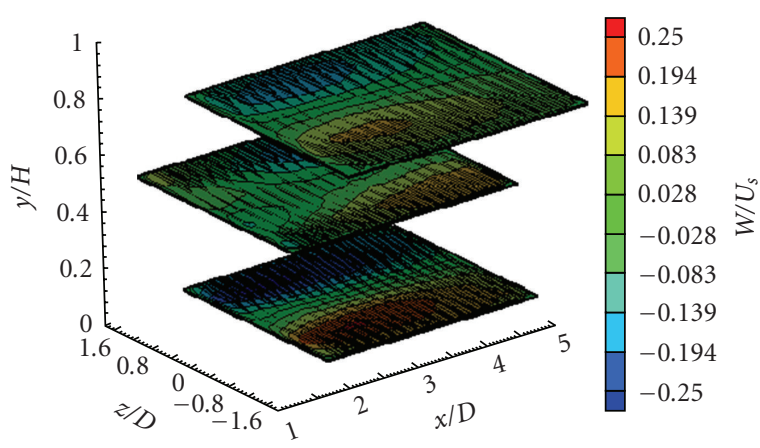

FIGURE 7: A vector plot representing the mean-flow field in the three horizontal planes $(y / H=0.10,0.51$ and 0.76$)$ superimposed by the contour of transverse velocity component. This figure clearly proves the vertical variability of the shallow wake. In this figure $W$ represents velocity in $z$-direction.

parameters on the uniqueness of shallow wake have been discussed. The origin and development of 2DCS have been reviewed.

Based on the review of the previous literature, a probable model of the flow geometry downstream of a bluff body immersed in a shallow open channel flow can be hypothesized as follows: the vertically sheared approaching flow, incident on the body, encounters the horizontal shear due to the presence of the body. This will generate a typical horseshoe vortex which wraps around the body (effect of bed) and extends in the downstream direction. A typical signature of the horseshoe vortex can be found in $\mathrm{Fu}$ and Rockwell [60] based on the Reynolds stress contour downstream of the body close to the bed. Due to the presence of the horseshoe vortex, one would expect a pair of vortices (clockwise for negative $z$ and counterclockwise for positive $z$ ) close to the bed in the streamwise plane. Above these pair of vortices, the presence of two large vortices (counterclockwise for negative $z$, and vice versa) in the streamwise vertical plane ( $y-z$ plane behind the body) has been demonstrated by Eibeck [64] which induces an upward flow along the center of the body. Close to the body, within a few diameters downstream, the flow field in the near-wake region can be very dissipative due to intense interaction between the two above-mentioned vortices. However, the legs of the horseshoe vortices will dissipate faster than the other vortices, and then only the second pair of vortices will remain dominant through the entire flow depth. Close to the bed, along the centerline downstream of the body, the flow travels towards the saddle point and then upwards towards the free-surface. This particular axial flow from the nearbed to free surface has been experimentally documented by Koochesfahani [65]. When this flow reaches the vicinity of the free surface, it interacts with the turbulent events that originate near the free surface [14] and leads to energy redistribution [46] between the streamwise and transverse velocity components.

Based on the status of the present literature, the above flow picture starts to evolve, but there still exist missing links in the flow pattern. For example, the flow properties of the wake are different at different elevations from the bed and these properties can be expressed as a function of the distance from the bed, or some dimensionless numbers, which may take into account the damping effect of the bed, or the wall-effect of the free-surface. The unique nature of the vortex shedding for shallow wake, including the effect of the near-bed horseshoe vortex and the axial flow from the bed towards the free surface, has not been addressed in sufficient detail. The examination of the instantaneous streamline topology as a function of the distance of the bed may lead to the possibility of defining a common feature of the topology with variable distance from the bed. Most notable is the appearance of the 2DCS in the shallow wake flow, and the investigation of their characteristics is limited. A robust method of identifying and extracting these 2DCS from the flow is lacking. Once, successfully identified, the effect of the dynamics of these 2DCS on the shallow wake can be addressed in a clearer way.

\section{References}

[1] H. J. D. Vriend, "Complex flows in civil and environmental engineering," in Shallow Flows, G. H. Jirka and W. S. J. Uijttewaal, Eds., Taylor and Francis, London, UK, 2004.

[2] G. H. Jirka and W. S. J. Uijttewaal, Shallow Flows, Taylor \& Francis, London, UK, 2004.

[3] R. S. Scorer, Experimental Aerodynamics, Ellis Horwood Limited, Chichester, UK, 1978.

[4] R. E. Falco, "Coherent motions in the outer region of turbulent boundary layers," Physics of Fluids, vol. 20, no. 10, pp. S124S132, 1977.

[5] M. R. Head and P. Bandyopadhyay, "New aspects of turbulent boundary-layer structures," Journal of Fluid Mechanics, vol. 107, pp. 297-338, 1981.

[6] S. K. Robinson, "Coherent motions in the turbulent boundary layer," Annual Review of Fluid Mechanics, vol. 23, no. 1, pp. 601-639, 1991.

[7] R. J. Adrian, "Hairpin vortex organization in wall turbulencea," Physics of Fluids, vol. 19, no. 4, Article ID 041301, 2007.

[8] R. F. Blackwelder and L. S. G. Kovasznay, "Time scales and correlations in a turbulent boundary layer," Physics of Fluids, vol. 15 , no. 9 , pp. 1545-1554, 1972.

[9] T. Theodorsen, "The structures of turbulence," in 50th Jahre Grenzschictforschubg, H. Gortier and W. Tolmien, Eds., Friedrich Vieweg \& Sohn, Braunschweig, Germany, 1995.

[10] S. J. Kline and S. K. Robinson, "Quasi-coherent structures in the turbulent boundary layer-part I: status report on a community wide summery of the data," in Near Wall Turbulence, S. J. Kline and S. H. Afgan, Eds., Hemishpere, New York, NY, USA, 1989.

[11] Y. Wu and K. T. Christensen, "Spatial structure of a turbulent boundary layer with irregular surface roughness," Journal of Fluid Mechanics, vol. 655, pp. 380-418, 2010.

[12] R. J. Volino, M. P. Schultz, and K. A. Flack, "Turbulence structure in rough- and smooth-wall boundary layers," Journal of Fluid Mechanics, vol. 592, pp. 263-293, 2007.

[13] J. C. Lin, M. Ozgoren, and D. Rockwell, "Space-time development of the onset of a shallow-water vortex," Journal of Fluid Mechanics, vol. 485, pp. 33-66, 2003.

[14] V. Roussinova, A. M. Shinneeb, and R. Balachandar, "Investigation of fluid structures in a smooth open-channel flow 
using proper orthogonal decomposition," Journal of Hydraulic Engineering, vol. 136, no. 3, pp. 143-154, 2010.

[15] K. Satoru, U. Hiromasa, O. Fumimaru, and M. Tokuro, "Turbulence structure and transport mechanism at the free surface in an open channel flow," International Journal of Heat and Mass Transfer, vol. 25, no. 4, pp. 513-521, 1982.

[16] C. E. Willert and M. Gharib, "The interaction of modulated vortex pairs with free surface," in Proceedings of the ASME Symposium of Free-Surface Turbulence, FED, vol. 101, pp. 2536, ASME, June 1994.

[17] P. Maheo, Free surface turbulent shear flow, Ph.D. dissertation, California Institute of Technology, 1999.

[18] J. C. R. Hunt and J. M. R. Graham, "Free stream turbulence in near plane boundaries," Journal of Fluid Mechanics, vol. 84, no. 2, pp. 209-235, 1978.

[19] D. T. Walker, R. I. Leighton, and L. O. Garza-Rios, "Shear-free turbulence near a flat free surface," Journal of Fluid Mechanics, vol. 320, pp. 19-51, 1996.

[20] R. Balachandar and V. C. Patel, "Velocity measurements in a developed open channel flow in the presence of an upstream perturbation," Journal of Hydraulic Research, vol. 43, no. 3, pp. 258-266, 2005.

[21] D. G. Dommermuth, "Initialization of vortical free-surface flows," Journal of Fluids Engineering, vol. 116, no. 1, pp. 95102, 1994.

[22] C. Zhang, L. Shen, and D. K. P. Yue, "The mechanism of vortex connection at a free surface," Journal of Fluid Mechanics, vol. 384, pp. 207-241, 1999.

[23] A. Weigard and M. Gharib, "A plane mixing layer with crossshear," Journal of Fluids Engineering, vol. 117, p. 374, 1995.

[24] K. Lam and S. Banerjee, "On the condition of streak formation in a bounded turbulent flow," Physics of Fluids A, vol. 4, no. 2, pp. 306-320, 1992.

[25] S. Komori, R. Nagaosa, Y. Murakami, S. Chiba, K. Ishii, and K. Kuwahara, "Direct numerical simulation of threedimensional open-channel flow with zero-shear gas-liquid interface," Physics of Fluids A, vol. 5, no. 1, pp. 115-125, 1993.

[26] R. A. Handler, T. F. Swean Jr., R. I. Leighton, and J. D. Swearingen, "Length scales and the energy balance for turbulence near a free surface," AIAA Journal, vol. 31, no. 11, pp. 1998-2007, 1993.

[27] Y. Pan and S. Banerjee, "A numerical study of free-surface turbulence in channel flow," Physics of Fluids, vol. 7, no. 7, pp. 1649-1664, 1995.

[28] R. Gupta, S. Kumar, and S. Banerjee, "Characteristics of attached vortices in free surface channel flows," in Proceedings of the 12th US National Congress of Applied Mechanics, 1994.

[29] V. Nikora, R. Nokes, W. Veale, M. Davidson, and G. H. Jirka, "Large-scale turbulent structure of uniform shallow free-surface flows," Environmental Fluid Mechanics, vol. 7, no. 2, pp. 159-172, 2007.

[30] L. Shen, X. Zhang, D. K. P. Yue, and G. S. Triantafyllou, "The surface layer for free-surface turbulent flows," Journal of Fluid Mechanics, vol. 386, pp. 167-212, 1999.

[31] M. Zdravkovich, Flow around Circular Cylinder, vol. 1, Oxford University Press, New York, NY, USA, 1997.

[32] M. Van Dyke, An Album of Fluid Motions, Parabolic Press, Stanford, Calif, USA, 1982.

[33] S. Barbarutsi and V. H. Chu, "Experimental study of turbulent mixing layers in shallow open channel flow," Tech. Rep. 85-1, McGill University, 1985.
[34] V. H. Chu, J. H. Wu, and R. E. Khayat, "Stability of turbulent shear flows in shallow channel," in Proceedings of the 20th IAHR Congress, vol. 3, Moscow, Russia, 1983.

[35] E. Wolonski, J. Imberger, and M. L. Heron, "Island wakes in shallow coastal waters," Journal of Geophysical Research, vol. 89, pp. 10553-10569, 1984.

[36] R. G. Ingram and V. H. Chu, "Flow around islands in Rupert Bay: an investigation of the bottom friction effect," Journal of Geophysical Research, vol. 92, no. 13, pp. 14521-14533, 1987.

[37] D. Chen and G. H. Jirka, "Experimental study of plane turbulent wakes in a shallow water layer," Fluid Dynamics Research, vol. 16, no. 1, pp. 11-41, 1995.

[38] M. E. Negretti, S. A. Socolofsky, A. C. Rummel, and G. H. Jirka, "Stabilization of cylinder wakes in shallow water flows by means of roughness elements: an experimental study," Experiments in Fluids, vol. 38, no. 4, pp. 403-414, 2005.

[39] D. T. Walker and V. G. Johnston, "Observation of turbulence near the free surface in the wake of a model ship," in Dynamics of Bubbles and Vortices Near a Surface, I. Sahin and G. Tryggvason, Eds., AMD, vol. 119, ASME, New York, NY, USA, 1991.

[40] D. G. Anthony and W. W. Willmarth, "Turbulence measurements in a round jet beneath a free surface," Journal of Fluid Mechanics, vol. 243, pp. 699-720, 1992.

[41] L. M. Logory, A. Hirsa, and D. G. Anthony, "Interaction of wake turbulence with a free surface," Physics of Fluids, vol. 8, no. 3, pp. 805-815, 1996.

[42] S. K. Rao, D. Sumner, and R. Balachandar, "A visualization study of fluid-structure interaction between a circular cylinder and a channel bed," Journal of Visualization, vol. 7, no. 3, pp. 187-199, 2004.

[43] H. F. Wang, Y. Zhou, C. K. Chan, and K. S. Lam, "Effect of initial conditions on interaction between a boundary layer and a wall-mounted finite-length-cylinder wake," Physics of Fluids, vol. 18, no. 6, Article ID 065106, 2006.

[44] T. Dracos, M. Giger, and G. H. Jirka, "Plane turbulent jets in a bounded fluid layer," Journal of Fluid Mechanics, vol. 241, pp. 587-614, 1992.

[45] W. S. J. Uijttewaal and J. Tukker, "Development of quasi twodimensional structures in a shallow free-surface mixing layer," Experiments in Fluids, vol. 24, no. 3, pp. 192-200, 1998.

[46] A. Singha, A.-M. Shinneeb, and R. Balachandar, "PIV-POD investigation of the wake of a sharp-edged flat bluff body immersed in a shallow channel flow," Journal of Fluids Engineering, vol. 131, no. 2, Article ID 021202, 2009.

[47] P. Holmes, J. L. Lumley, and G. Berkooz, Turbulence, Coherent Structures, Dynamical Systems and Stability, Cambridge University Press, 1996.

[48] J. P. Bonnet, J. Delville, M. N. Glauser et al., "Collaborative testing of eddy structure identification methods in free turbulent shear flows," Experiments in Fluids, vol. 25, no. 3, pp. 197-225, 1998.

[49] A. K. M. F. Hussain, "Coherent structures—reality and myth," Physics of Fluids, vol. 26, no. 10, pp. 2816-2850, 1983.

[50] C. F. V. Carmer and G. H. Jirka, "On turbulent and transport in shallow wake flows," in Proceedings of the IAHR Conference, Beijing, China, 2001.

[51] G. H. Jirka, "Large scale flow structures and mixing processes in shallow flows," Journal of Hydraulic Research, vol. 39, no. 6, pp. 567-573, 2001.

[52] R. H. Kraichnan, "Inertial ranges in two-dimensional turbulence,” Physics of Fluids, vol. 10, no. 7, pp. 1417-1423, 1967. 
[53] R. Balachandar, M. F. Tachie, and V. H. Chu, "Concentration profiles in shallow turbulent wakes," Journal of Fluids Engineering, vol. 121, no. 1, pp. 34-43, 1999.

[54] E. Wolansky, "Water circulation in a topographically complex environment," in Proceedings of the International Symposium on the Physics of Shallow Estuaries and Bays, University of Miami, 1984.

[55] M. F. Tachie, Scaler transport in Intermediate two-dimensional turbulent wakes, M.S. thesis, University of Saskatchewan, 1997.

[56] R. Balachandar, S. Ramachandran, and M. F. Tachie, "Characteristics of shallow turbulent near wakes at low Reynolds numbers," Journal of Fluids Engineering, vol. 122, no. 2, pp. 302-308, 2000.

[57] M. F. Tachie and R. Balachandar, "Shallow wakes generated on smooth and rough surfaces," Experiments in Fluids, vol. 30, no. 4, pp. 467-474, 2001.

[58] H. Akilli and D. Rockwell, "Vortex formation from a cylinder in shallow water," Physics of Fluids, vol. 14, no. 9, pp. 2957 2967, 2002.

[59] H. Fu and D. Rockwell, "Shallow flow past a cylinder: transition phenomena at low Reynolds number," Journal of Fluid Mechanics, vol. 540, pp. 75-97, 2005.

[60] H. Fu and D. Rockwell, "Shallow flow past a cylinder: control of the near wake," Journal of Fluid Mechanics, vol. 539, pp. 124, 2005.

[61] R. K. Cohn and M. M. Koochesfahani, "Effect of boundary conditions on axial flow in a concentrated vortex core," Physics of Fluids A, vol. 5, no. 1, pp. 280-282, 1993.

[62] A. E. Perry and T. R. Steiner, "Large scale vortex structures in turbulent wakes behind bluff bodies: 1 . vortex formation process," Journal of Fluid Mechanics, vol. 174, pp. 233-270, 1987.

[63] A. Singha, Shallow wake in open channel flow-a look into the vertical variability, Ph.D. dissertation, University of Windsor, Windsor, Canada, 2009, http://winspace.uwindsor. ca/wintheses/singha2.pdf.

[64] P. A. Eibeck, "An experimental study of the flow downstream of a circular and tapered cylinder," Journal of Fluids Engineering, vol. 112, no. 4, pp. 393-401, 1990.

[65] M. M. Koochesfahani, "Vortical patterns in the wake of an oscillating airfoil," AIAA Journal, vol. 27, no. 9, pp. 1200-1205, 1989. 

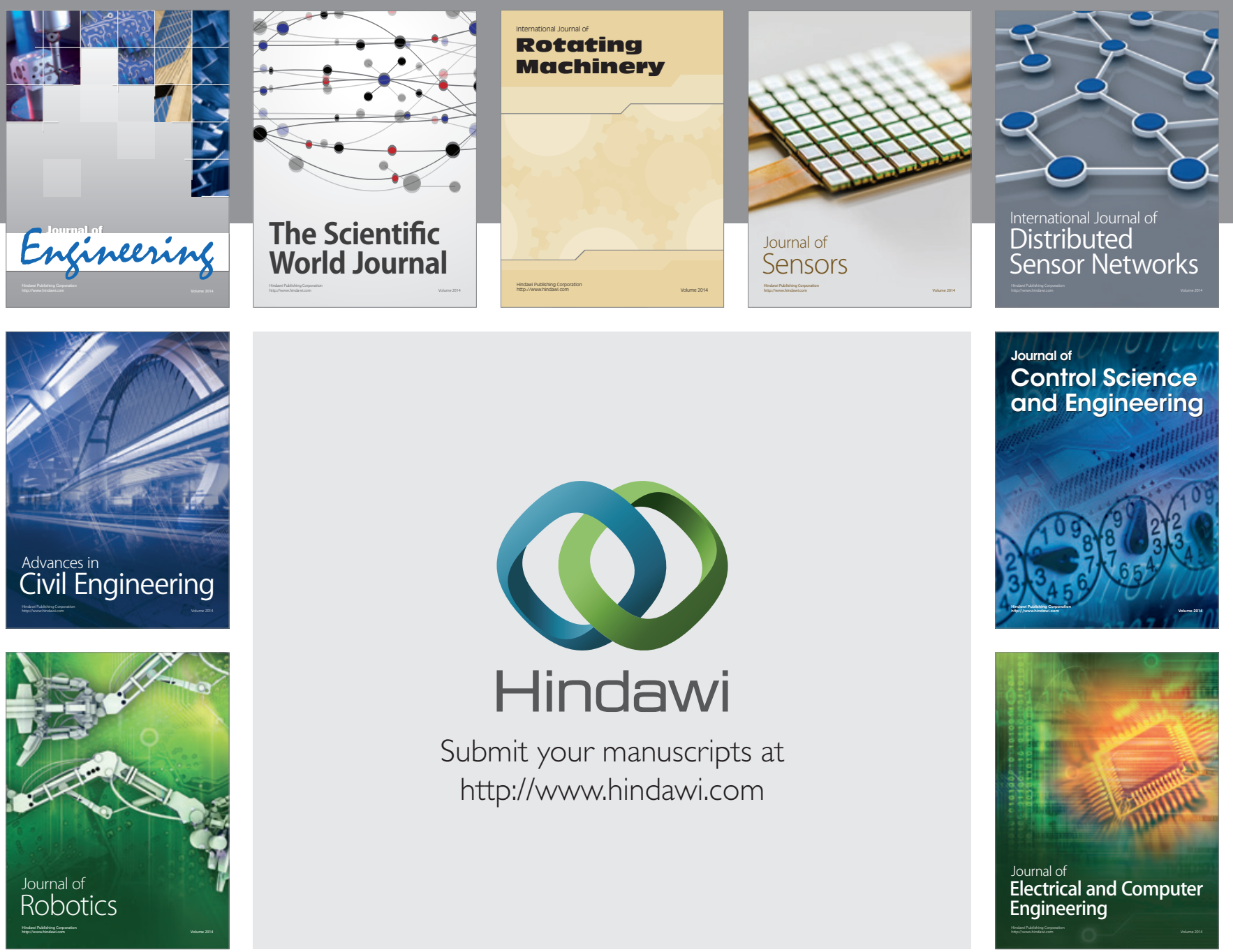

Submit your manuscripts at

http://www.hindawi.com
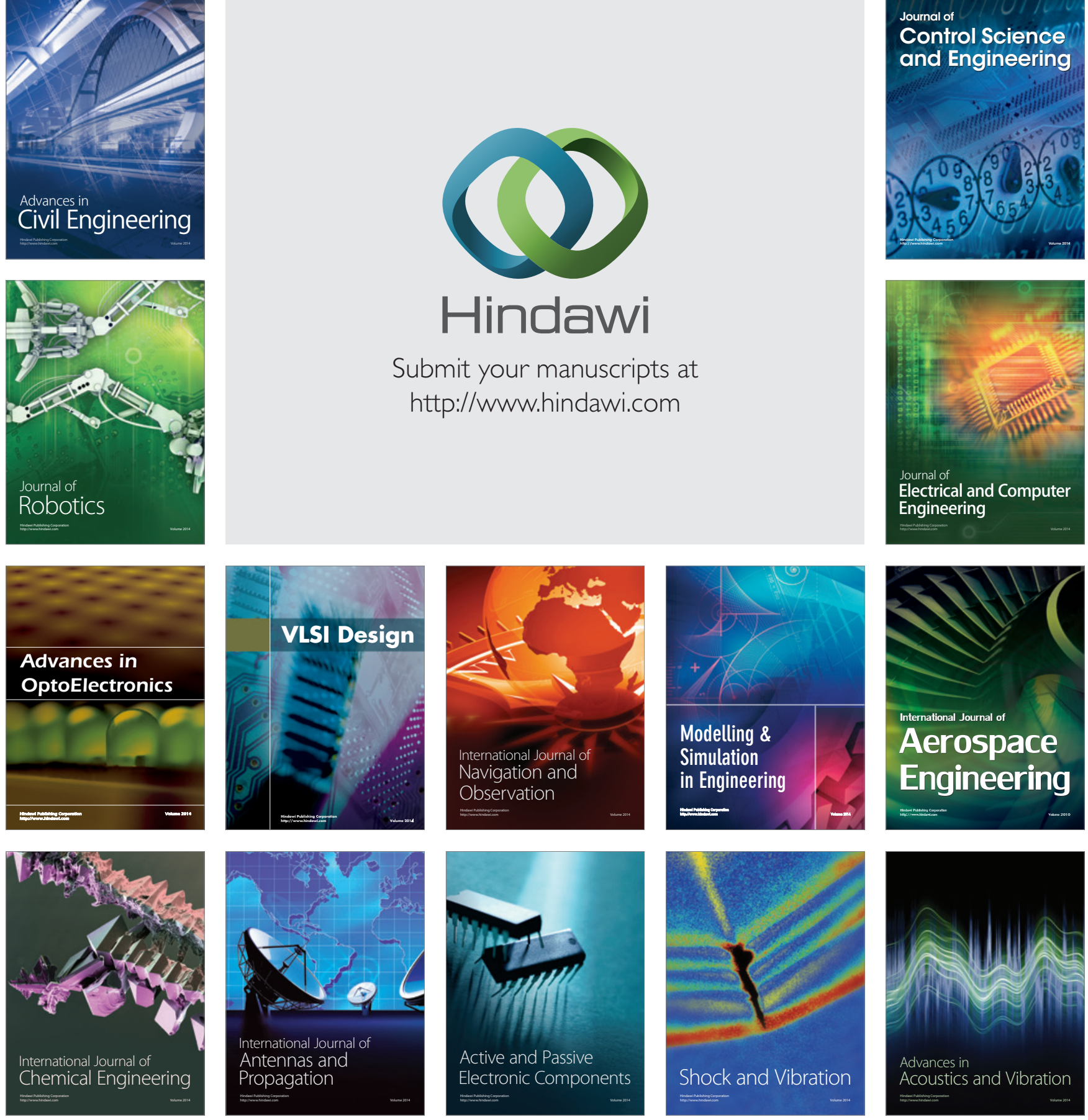